\title{
Diabetic retinopathy in Victoria, Australia: the Visual Impairment Project
}

\author{
Robert McKay, Catherine A McCarty, Hugh R Taylor
}

\begin{abstract}
Aim-To establish the prevalence, severity, and risk factors for diabetic retinopathy in a representative sample of Victorian residents aged 40 years and older.

Methods-A population based, cluster sampling method was used to recruit 4744 participants $(86 \%$ participation rate). Nine randomly selected, suburban Melbourne clusters and four randomly selected, rural Victorian clusters were used. Participants provided a detailed medical and personal history and underwent an ocular examination including funduscopy and fundus photography. Rural participants provided a blood sample, from which the glycosylated haemoglobin percentage was measured. The diagnosis of diabetic retinopathy was based on fundus photographs from participants with self reported diabetes.
\end{abstract}

Results - The prevalence of diabetic retinopathy among people with self reported diabetes was $29.1 \%$. The prevalence of untreated, vision threatening retinopathy was $2.8 \%$. Retinopathy was positively associated with a longer reported duration of diabetes diagnosis $(p<0.01)$ and with higher fractions of glycosylated haemoglobin $(p<0.01)$. Retinopathy was not significantly associated with age, ethnicity, body mass index, glaucoma, myopia or intake of alcohol, tobacco, or aspirin (all p $>0.05$ ).

Conclusions-Most people in Victoria with proliferative diabetic retinopathy or clinically significant macular oedema have received laser treatment. There remains however, a small but important group who have not received treatment and whose vision is threatened. People with diabetes should be encouraged to maintain strict glycaemic control and to undergo regular screening to delay or prevent the development of retinopathy. (Br f Ophthalmol 2000;84:865-870)

Diabetic retinopathy is the most common chronic complication of diabetes ${ }^{1}$ and one of the most devastating. In the USA it has been estimated that diabetic retinopathy (DR) is the most common cause of new cases of blindness in people aged $25-74$ years. ${ }^{1}$

The Visual Impairment Project (VIP) ${ }^{2}$ is a large, population based epidemiological study which is ongoing in the state of Victoria, Australia. It has been designed to investigate the distribution and determinants of eye disease, the impact of blindness and visual impairment on individuals, and the use of and barriers to eye healthcare services. This report analyses the epidemiology of DR using cross sectional data collected during the baseline phase of the project between 1992 and 1996.

We report the prevalence of DR, based on fundus photographs from participants with self reported diabetes. This includes an evaluation of the prevalence of vision threatening DR as well as previous laser treatment for DR. We also demonstrate in our cohort, the well established $^{3}$ association between both duration and control of diabetes and the prevalence of retinopathy.

\section{Methods}

A stratified cluster sampling method was used to select participants. Each cluster consisted of two adjacent census collector districts. The urban component of the project contained nine randomly selected Melbourne suburban clusters. The rural component contained four randomly selected clusters from throughout the rest of the state.

A private household census was conducted to identify permanent household members aged 40 years or older who were classified as eligible residents. A screening questionnaire was then administered in the home to establish contact with eligible residents and to determine basic demographics. When respondents were not available, repeated visits were made to the home. Eligible residents were then invited to attend a local temporary test site for a more comprehensive interview and an ophthalmic examination. Those who were unable to attend the test site were offered a modified home visit so that as much information as possible could be obtained.

At the test site, another interview was conducted by trained interviewers. It elicited further demographic details, a history of medical and ocular health, a history of smoking and alcohol intake, and a history of medication use. Participants were classified into various ethnic groups on the basis of their report of their parents' country of birth. Body weight and height were questioned and body mass index (BMI) was calculated as weight $(\mathrm{kg}) /\left(\right.$ height $\left.(\mathrm{m})^{2}\right)$.

The interview included specific questioning about whether participants had ever been diagnosed with diabetes mellitus and the year in which such a diagnosis was made. Participants with previously diagnosed diabetes were also asked if and when their last dilated fundus examination had been conducted and who performed the examination. The questions relating to diabetes have been previously employed and validated by Welborn et al. ${ }^{4}$ 
Participants underwent a detailed examination of each eye. Pupils were dilated with one drop of tropicamide $(0.5 \%)$ and one drop of phenylephrine hydrochloride (10\%). Stereoscopic fundus photographs were then taken with a Topcon TRC fundus camera (Topcon, Paramus, NJ, USA). Two 30 degree fields of the fundus were photographed in each eye; one centred on the optic disc and the other centred on the fovea. Photographs from participants who reported having diabetes were evaluated by an ophthalmologist with medical retina subspecialist qualifications. This evaluation was used to establish the presence or absence of any diabetic retinopathy (DR), proliferative retinopathy, clinically significant macular oedema (as defined by the Early Treatment Diabetic Retinopathy Study (ETDRS) ${ }^{5}$ ), and previous retinal laser treatment. Levels of diabetic retinopathy were defined, according to the Academy of Ophthalmology in the United States, as mild non-proliferative, moderate non-proliferative, severe non-proliferative, and proliferative $\mathrm{DR}^{6}{ }^{6}$

Dilated funduscopy was performed by an ophthalmologist with a 90 dioptre hand held fundus viewing lens used at a Topcon SL slit lamp (Topcon). This was supplemented by indirect ophthalmoscopy if peripheral abnormalities were revealed or suspected. The presence or absence of any DR, proliferative retinopathy, clinically significant macular oedema, or previous retinal laser treatment was recorded.

In this report, DR status is assessed only in participants with self reported diabetes. Assignment of DR status is based on fundus photographs where they are available. In 14 participants for whom fundus photographs were not available, the findings from dilated funduscopy have been used to determine DR status. Two participants who reported having diabetes refused fundus photography and fundus examination but allowed us to obtain information about DR status from their ophthalmologist.

All participants at rural test sites were asked to provide a fingerprick blood sample. This was used to measure glycosylated haemoglobin percentages $\left(\mathrm{HbA}_{1 \mathrm{c}}\right)$, via a Bayer DCA2000 $\mathrm{HbA}_{1 \mathrm{c}}$ analyser (Bayer Co, Elkhart, IN, USA). This device has a measurable range of $\mathrm{HbA}_{10}$ from $3.9 \%$ to $14.0 \%$. A reading of $14.0 \%$ was found in three participants who all reported a diagnosis of diabetes and was entered as the actual level. A reading of $3.9 \%$ was found in one participant who reported no diagnosis of diabetes and was entered as the actual level.

Glaucoma status was determined by the following method. Intraocular pressure was measured with a hand held applanation tonometer (Oculab Tonopen, Oculab, La Jolla, CA, USA). In participants with Tonopen readings $\geqslant 22 \mathrm{~mm} \mathrm{Hg}$ in either eye, pressure was checked with Goldmann applanation tonometry. A Humphrey computerised visual field analyser (Humphrey Instruments, Dublin, CA, USA) was used to record 24-2 FASTPAC visual fields in all participants. Participants who were unable reliably to complete computerised visual field assessments underwent Bjerrum visual field testing. Glaucoma status was evaluated by a consensus panel comprising six ophthalmologists, including two glaucoma specialists. This panel determined glaucoma status on the basis of Humphrey visual fields and photographs of the optic disc. All participants with a cup/disc ratio that was enlarged $(\geqslant 0.7)$ or asymmetrical ( $\geqslant 0.3$ interocular difference), a glaucomatous visual field defect, or an intraocular pressure of greater than $21 \mathrm{~mm} \mathrm{Hg}$ in either eye were presented to the panel. ${ }^{7}$

The presence of myopia was determined as follows. Presenting visual acuity was determined with an ETDRS 4 metre chart. Any spectacle correction worn was measured with a Humphrey automatic lens analyser (model 330, Humphrey Instruments, Dublin, CA, USA). A Humphrey automatic refractor (Humphrey Instruments) was used to measure the objective refraction in all participants who read fewer than 53 letters (6/7.5 or $20 / 25)$ in either eye. This was then refined by participative refraction. Myopia was defined as a best corrected minus spherical equivalent of more than -0.5 dioptres in either eye.

Double entry of data with comparison was used to minimise errors in the data set. Statistical analyses were performed using SAS version 6.09 (SAS Institute Inc, Cary, NC, USA). The statistical procedures used in this research include summary statistics, $t$ tests, $\chi^{2}$ analyses, and multiple logistic regression. These procedures are documented in the SAS/STAT Users' Guide. $^{8}$ A p value of $<0.05$ was considered to be statistically significant.

The Royal Victorian Eye and Ear Hospital human research and ethics committee approved the project. Informed consent was obtained from all participants.

Further details about the methodology of the urban component of the VIP are published elsewhere. ${ }^{2}$ For the purposes of this report, the sampling and study methods used in the rural component of the VIP were the same except that glycosylated haemoglobin measurements were added. A comparison of participants with non-participants from the urban component of the VIP has also been published. ${ }^{9}$

\section{Results}

In total, 5520 participants were identified as being eligible to participate in the rural and urban components of the VIP. Of these, 776 did not participate, giving a response rate of $86 \%$. In the urban clusters, the overall response rate was $84 \%$ (3271/3912). In the rural clusters, the overall response rate was $92 \%(1473 / 1608)$.

The prevalence of self reported diabetes was $5.1 \%$ (239/4478). This did not differ significantly between the urban and the rural samples $(5.2 \%$ and $4.9 \%$ respectively, $\mathrm{p}=0.69)$. The number of participants who reported using medications to control their diabetes was 172 $(72 \%)$. There were 39 participants who used insulin (16.6\%) and 133 participants who used oral hypoglycaemic agents $(56.6 \%)$. This includes four participants who reported using both insulin and oral hypoglycaemics. A 
further four participants reported using medications for diabetes control but did not report what these medications were.

We have analysed the fundus photographs from both eyes of 215 of the 239 people in our cohort who reported a diagnosis of diabetes. In three participants there was a photograph of one eye only, which showed evidence of DR. In 14 of the remaining participants without photographs, information was available from indirect ophthalmoscopy. For two participants there were no photographs or funduscopy but information was available from a treating ophthalmologist that DR was present in at least one eye. Thus, we have reliable information about the presence or absence of DR in either eye from 234 of the 239 people with self reported diabetes.

The prevalence of DR among people with self reported diabetes was $32.3 \%(53 / 164)$ in the urban sample and $21.4 \%(15 / 70)$ in the rural sample. As this difference was not statistically significant $(p=0.10)$, we have combined information from the two samples. This gives an overall prevalence of DR among people with self reported diabetes of $29.1 \%$ (68/234).

The prevalence of DR in men was $29.5 \%$ (35/135), which was not significantly different from the prevalence in women of $33.3 \%$ $(33 / 99)(p=0.22)$. The mean age of participants with DR was 64.2 years (median 63.5, range 45-91). The mean age of participants with self reported diabetes but without DR was 64.6 years (median 65.0 , range $42-84$ ). The prevalence of DR did not vary significantly with age $(\mathrm{p}=0.22)$.

The prevalence of DR in participants who reported a diabetes diagnosis before the age of 30 was $50.0 \%$ (4/8). The prevalence of DR in participants who reported a diabetes diagnosis at the age of 30 or older was $28.3 \%(64 / 226)$. This difference was not statistically significant $(\mathrm{p}=0.18)$.

The mean reported duration of diabetes diagnosis in participants with DR was 14.6 years (median 14.0, range 0-44). The mean reported duration of diabetes diagnosis in participants without DR was 6.8 years (median 5.0, range $0-57$ ). This difference was statistically significant $(\mathrm{p}<0.01)$. Figure 1 summa-

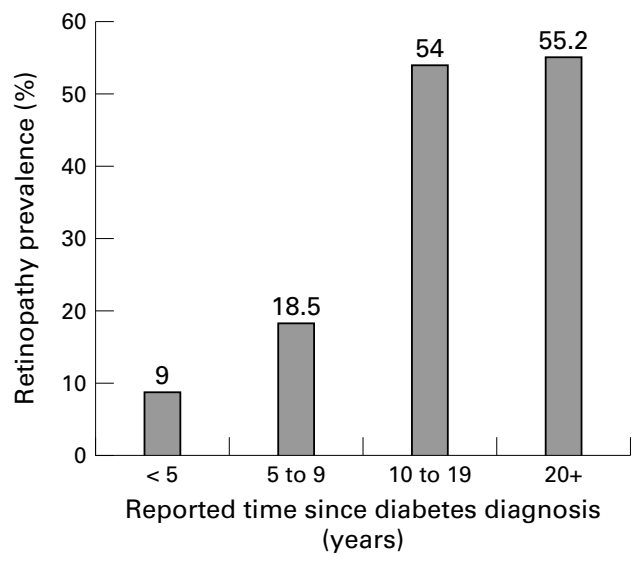

Figure 1 Prevalence of retinopathy by reported duration of diabetes diagnosis.

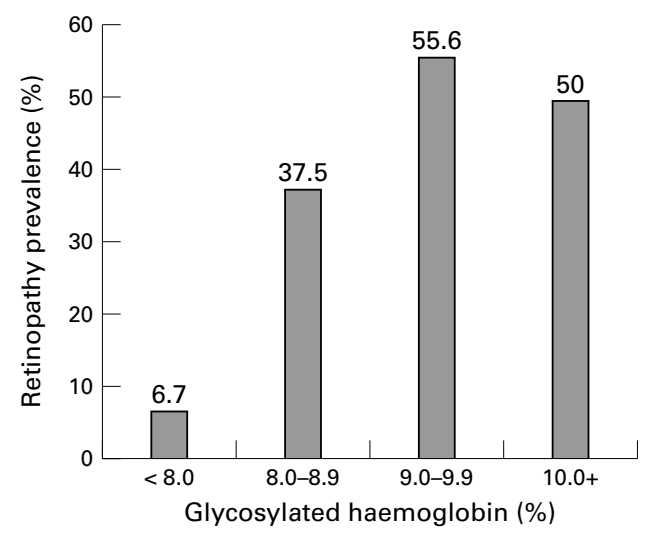

Figure 2 Prevalence of retinopathy by glycosylated haemoglobin level.

rises DR prevalence in different categories of reported diabetes duration.

We have glycosylated haemoglobin measurements for 70 participants with self reported diabetes, 15 of whom had diabetic retinopathy. The mean $\mathrm{HbA}_{1 \mathrm{c}}$ in participants with $\mathrm{DR}$ was $9.3 \%$ (median 9.1 , range $7.0-14.0$ ). The mean $\mathrm{HbA}_{1 \mathrm{c}}$ in participants without DR was $7.2 \%$ (median 6.9, range 4.7-14.0). This difference was statistically significant $(\mathrm{p}<0.01)$. Figure 2 summarises DR prevalence in different categories of $\mathrm{HbA}_{1 \mathrm{c}}$ measurement.

The prevalence of DR in participants who reported using insulin was $67.6 \%$ (25/37). The prevalence of DR in those who reported using oral hypoglycaemic agents without insulin to control their diabetes was 29.9\% (38/127). The prevalence of DR in participants who reported using no diabetes medications was $7.5 \%(5 / 67)$.

Among people with self reported diabetes, no statistically significant difference could be found between participants with DR and those without DR in terms of ethnicity, body mass index, glaucoma, myopia, alcohol intake, tobacco intake, aspirin use (current), or antihypertensive use (current or ever) (data not presented).

To examine the treatment and severity of DR, we only included the 215 participants with self reported diabetes for whom we had graded fundus photographs from both eyes. There were 14 participants with evidence of laser treatment $(6.5 \%)$ and this was bilateral in nine of these $(4.2 \%)$. There were 12 people who had clinically significant macular oedema $(5.6 \%)$ and this was bilateral in five of these $(2.3 \%)$. Untreated clinically significant macular oedema was found in five participants, including one person with bilateral untreated clinically significant macular oedema and one person with treated macular oedema in the fellow eye. There were nine people with proliferative DR (4.2\%) and this was bilateral in five of these $(2.3 \%)$. Only one participant had untreated proliferative DR. This participant had treated proliferative DR in the other eye and did not have macular oedema. Combining this information gives six people $(2.8 \%)$ with untreated, vision threatening DR including one person with bilateral, untreated, vision threatening DR (0.47\%). The prevalence of mild, 
moderate, and severe non-proliferative DR was $16.7 \%(\mathrm{n}=37), 6.8 \%(\mathrm{n}=15)$, and $0.5 \%(\mathrm{n}=1)$, respectively.

The duration of diabetes diagnosis in the 12 participants with clinically significant macular oedema ranged from 4 years to 30 years with a median of 17.5 years and a mean of 17.7 years. The duration of diabetes diagnosis in the nine participants with proliferative DR ranged from 10 years to 38 years with a median of 18 years and a mean of 20.1 years.

One hundred and thirty one participants $(64 \%)$ reported having had a dilated fundus examination at least once. Participants taking insulin were far more likely to have ever had a retinal examination $\left(91 \%\right.$ versus $59 \%, \chi^{2}$ $=12.4, \mathrm{p}=0.001)$. In a multivariate logistic regression model with backwards selection that included age, sex, years since diabetes diagnosis, insulin treatment, born outside Australia, and English spoken at home, the following variables were found to be significantly associated with never having had a retinal examination: born outside Australia (OR=2.24, $95 \% \mathrm{CL}=1.23,4.08)$ and not treated with insulin $(\mathrm{OR}=6.67,95 \% \mathrm{CL}=1.93,22.7)$.

One hundred and ninety five participants could recall the year of their last retinal examination. Of them, the percentage who had a retinal examination within the past 2 years by level of DR was: none, $49.3 \%$; mild nonproliferative retinopathy, $41.4 \%$; moderate non-proliferative retinopathy, $83.3 \%$; severe non-proliferative retinopathy, $0 \%$; proliferative retinopathy, 55.6\%. Participants who had any DR were not more likely to have had a retinal examination in the previous 2 years than participants without DR $(52.9 \%$ versus $\left.49.3 \%, \chi^{2}=0.20, p=0.66\right)$. People treated with insulin were significantly more likely to have had a dilated fundus examination in the previous 2 years than people treated with diet and $/$ or tablets $\left(69.7 \%\right.$ versus $48.0 \%, \chi^{2}=5.24$, $\mathrm{p}=0.02$ ).

Among participants who reported no previous diagnosis of diabetes, there were no signs of DR found in any individual at the clinical eye examination.

\section{Discussion}

Our estimate of the prevalence of DR in people with self reported diabetes is $29 \%$. This is similar to the prevalence found in other population based studies. The Blue Mountain Eye Study $(\mathrm{BMES})^{10}$ found a DR prevalence of $32 \%$ among 253 people with diabetes aged 49 or older in western Sydney. This estimate included 38 people who were newly diagnosed with diabetes by the study group (fasting blood glucose $\geqslant 7.8$ ). The largest Australian study of DR to date is the Newcastle Diabetic Retinopathy Study. ${ }^{11}$ This was a longitudinal, clinic based study of people with diabetes of all ages, which was conducted over an 11 year period. An overall $35 \%$ prevalence of any signs of DR was reported at the conclusion of the study. The Beaver Dam Eye Study (BDES), ${ }^{12}$ from Wisconsin, USA, examined a population aged from 43 to 84 years. Their estimate for DR prevalence in 435 people with diabetes was
$37 \%$. This included 49 people who were newly diagnosed with diabetes by the study group (using $\mathrm{HbA}_{1 \mathrm{c}}$ levels). The Rotterdam study ${ }^{13}$ was a population based investigation of people aged 55 years or older in Holland which included a glucose challenge test for participants who were not taking antidiabetes medications. Their estimate of the prevalence of DR among people with diabetes was $26 \%$. The Wisconsin Epidemiologic Study of Diabetic Retinopathy (WESDR) ${ }^{1}$ investigated people with diabetes of all ages who were identified by primary care physicians. They found DR prevalence rates of $71 \%$ (type 1 diabetes) and $39 \%$ (type 2 diabetes). This group has also published a summary of DR prevalence and incidence data from other population based studies conducted before 1989 .

Variation in the photographic methods used to identify DR may at least partially explain the variation in prevalence between these studies. The WESDR ${ }^{1}$ and Newcastle ${ }^{11}$ studies used all seven of the standard 30 degree fields originally described in the Diabetic Retinopathy Study (DRS). ${ }^{14}$ The $\mathrm{BMES}^{10}$ used fields 1 to 5 (inclusive) and the $\mathrm{BDES}^{12}$ used fields 1,2 , and 3. These studies all found slightly higher prevalence rates than ours, which were based on only two 30 degree fields. (Our fields were centred on the disc and on the macula and thus are equivalent to DRS standard fields 1 and 2 respectively). In contrast, the Rotterdam study, ${ }^{13}$ which found lower prevalence rates than ours, used only one 35 degree field, centred on the macula. Moss et al investigated the effect on sensitivity of DR detection when a smaller area of the retina is examined. ${ }^{15}$ Using all 7 DRS standard fields as their gold standard, they found $87 \%$ sensitivity for detecting any retinopathy when only fields 1 and 2 are used as in our study and 95\% sensitivity when only fields $1-4$ are used.

Our data indicate an association between longer duration of diabetes and increased prevalence of retinopathy. The WESDR has shown that non-proliferative retinal lesions, macular oedema, and proliferative DR each have a prevalence that is strongly associated with the duration of diabetes diagnosis. ${ }^{3} 1617$ Other large epidemiological studies have also supported such associations. ${ }^{10} 121819$ The Newcastle Diabetic Retinopathy Study reported that among participants with diabetes but without retinopathy, $8 \%$ developed retinopathy each year. ${ }^{11}$

Our findings also demonstrate a correlation of lower $\mathrm{HbA}_{1 \mathrm{c}}$ levels with a lower prevalence of DR. An association between tighter diabetes control and lower DR prevalence has been consistently found in other large epidemiological studies. ${ }^{13} 182021$ The WESDR found that higher $\mathrm{HbA}_{1 \mathrm{c}}$ levels were positively associated with prevalence rates for non-proliferative lesions, proliferative $\mathrm{DR}$, and macular oedema and that $\mathrm{HbA}_{1 \mathrm{c}}$ predicts both the incidence and progression of retinopathy. ${ }^{3161722}$ The strongest available evidence comes from the Diabetes Control and Complications Trial (DCCT). ${ }^{23}$ This was a large, randomised clinical trial of participants with young onset insulin depend- 
ent diabetes mellitus (IDDM). It showed that the risk and progression of retinopathy can be reduced by intensive diabetic therapy, aiming to maintain near normal serum glucose levels. A $10 \%$ lower $\mathrm{HbA}_{1 \mathrm{c}}$ (for example, $8 \%$ versus $7.2 \%$ ) was associated with a $43 \%$ to $45 \%$ lower risk of progression of retinopathy. The study concluded that there was no level of $\mathrm{HbA}_{1 c}$ below which the risk of DR was completely eliminated. However, Warram et al used DCCT and WESDR data to suggest that in patients with IDDM, there may be a threshold value of $\mathrm{HbA}_{1 \mathrm{c}}$ of around $8.5 \%$, above which the risk of $\mathrm{DR}$ progression increases rapidly. ${ }^{24}$

There was a dramatic variation in DR prevalence according to the treatment method used to control diabetes. DR prevalence fell from $67.6 \%$ in participants who used insulin to $29.9 \%$ in participants who used oral medications without insulin to $7.5 \%$ in those who did not use medications. Duration of diabetes diagnosis and level of glycaemic control are both likely to be important confounding variables when examining treatment method. Unfortunately, we do not have the statistical power to examine treatment method with adjustment for duration and control.

Our study did not find any significant association between DR and age, ethnicity, $\mathrm{BMI}$, glaucoma, myopia, or intake of aspirin, alcohol, or tobacco. The ETDRS randomised participants to either placebo or aspirin and showed no effect of aspirin on the course of retinopathy..$^{25}$ The other variables listed have all been implicated as possibly being associated with DR but their significance remains unconfirmed. ${ }^{318}{ }^{26}$ The relatively small number of people with DR in our population based study does not give us the statistical power to identify weak associations.

It is a concern that only about half of people with diagnosed diabetes have had a retinal examination in the previous 2 years as recommended by the National Health and Medical Research Council clinical practice guidelines. ${ }^{27}$ We found previously that $34 \%$ of people without DR and $25 \%$ of people with DR in Melbourne had never seen an ophthalmologist. ${ }^{28}$ We also found that although the majority of general practitioners report that they do not perform dilated funduscopy, they usually refer their patients with diabetes to an ophthalmologist. ${ }^{29}$ Currently, a national diabetes strategy is being implemented in Australia. ${ }^{30}$ One of its aims is to reduce complications in people with diabetes, including diabetic retinopathy. A measure of the success of this implementation strategy will be an increase in the percentage of people with diabetes who have the recommended 2 yearly dilated fundus examination.

Currently, there are only two available methods for preventing blindness from retinopathy in people with diabetes. Firstly, glycaemic control should be kept as strict as possible so that the development and progression of retinopathy can be delayed. Blindness prevention should be discussed along with treatment of hypertension and hyperlipidaemia with patients as the strongest incentives to achieve strict control. Secondly, regular screening of people with diabetes is required to identify vision threatening retinopathy as early as possible so that timely laser treatment can be given. In Victoria, we have identified that there is a small but important number of people in the community with known diabetes who have untreated, vision threatening retinopathy. The necessity of regular screening must be emphasised to all people with diabetes so that this number is kept to an absolute minimum.

The Visual Impairment Project is supported by the Victorian Health Promotion Foundation (Vic Health), The Ansell Ophthalmology Foundation, The National Health and Medical Research Council Sir John Eccles award to Professor HR Taylor, the estate of the late Dorothy Edols, and the Jack Brockhoff Foundation. Dr McCarty is the recipient of the Wagstaff fellowship in ophthalmology from the Royal Victorian Eye and Ear Hospital.

1 Klein R, Klein BEK, Moss SE. The Wisconsin Epidemioogic Study of Diabetic Retinopathy: a review. Diab Met Rev 1989;5:559-70

2 Livingston PM, Carson CA, Stanislavsky YL, et al. Methods for a population-based study of eye disease: the Melbourne Visual Impairment Project. Ophthalmic Epidemiol 1994;1: $139-48$

3 Klein R, Klein BEK, Moss SE. Epidemiology of proliferative diabetic retinopathy. Diabetes Care 1992;15:1875-91. 4 Welborn TA, Knuiman MW, Bartholomew HC, et al.
1989-90 National Health Survey: prevalence of self reported diabetes in Australia. Med f A Aust 1995;163:12032 .

5 Early Treatment Diabetic Retinopathy Study Research Group. Grading diabetic retinopathy from stereoscopic color fundus photographs - an extension of the Modified Airlie House Classification. ETDRS Report Number 10. Ophthalmology 1991;98(Suppl):786-806.

6 American Academy of Ophthalmology. Preferred practice pattern: diabetic retinopathy. San Francisco: American Academy of Ophthalmology, 1989

7 Wensor MD, McCarty CA, Stanislavsky YL, et al. The prevalence of glaucoma in the Melbourne Visual Impairment Project. Ophthalmology 1998;105:733-9.

8 SAS/STAT User's Guide, Version 6. 4th ed. Vols 1 and 2. Cary, NC: SAS Institute Inc, 1989

9 Livingston PM, Lee SE, McCarty CA, et al. A comparison of participants with non-participants in a population-based epidemiologic study: the Melbourne Visual Impairment Project. Ophthalmic Epidemiol 1997;4:73-81.

10 Mitchell P, Smith W, Wang JJ, et al. Prevalence of diabetic retinopathy in an older community: the Blue Mountain Eye retinopathy in an older community: the B

11 Mitchell P, Moffitt P. Update and implications from the Newcastle diabetic retinopathy study. Aust NZ F Ophthalmol 1990;18:13-17.

12 Klein R, Klein BEK, Moss SE, et al. The Beaver Dam Eye Study: retinopathy in adults with newly discovered and previously diagnosed diabetes mellitus. Ophthalmology 1992;99:58-60

13 Stolk RP, Vingerling JR, Paulus TVM, et al. Retinopathy, glucose and insulin in an elderly population: the Rotterdam Study. Diabetes $1995 ; 44: 11-15$.

14 The Diabetic Retinopathy Study Group. A Modification of the Airlie House Classification of Diabetic Retinopathy. DRS report No 7. Invest Ophthalmol Vis Sci 1981;21:21026.

15 Moss SE, Meuer SM, Klein R, et al. Are seven standard photographic fields necessary for classification of diabetic photographic fields necessary for classification of diabet

16 Klein R, Klein BEK, Moss SE, et al. The Wisconsin Epidemiologic Study of Diabetic Retinopathy: VII. Diabetic miologic Study of Diabetic Retinopathy: VII. Diabetic
nonproliferative retinal lesions. Ophthalmology 1987;94: nonprolifer

17 Klein R, Klein BEK, Moss SE, et al. The Wisconsin Epidemiologic Study of Diabetic Retinopathy: IV. Diabetic macular edema. Ophthalmology 1984; 91:1464-74.

18 Ballard DJ, Melton LJ, Dwyer MS, et al. Risk factors for diabetic retinopathy: A population-based study in Rochester Minnesota. Diabetes Care 1986;9:334-42.

19 Orchard TJ, Dorman JS, Maser RE, et al. Prevalence of complications in IDDM by sex and duration. Pittsburgh Epidemiology of Diabetes Complications Study II. Diabetes 1990;39:1116-24.

20 Kingsley LA, Dorman JS, Doft BH, et al. An epidemiologic approach to the study of retinopathy: the Pittsburgh diabetic morbidity and retinopathy studies. Diab Res Clin Prac 1988;4:99-109.

21 Davis MD, Fisher MR, Gangnon RE, et al. Risk factors for high risk proliferative diabetic retinopathy and severe visual loss: Early Treatment Diabetic Retinopathy Study Report \# 18. Invest Ophthalmol Vis Sci 1998;39:233-52. 
22 Klein R, Klein BEK, Moss SE, et al. Glycosylated hemoglobin predicts the incidence and progression of diahemoglobin predicts the incidence and progress
betic retinopathy. $¥ A M A 1988 ; 260: 2864-71$.

23 The Diabetes Control and Complications Trial Research Group. The relationship of glycaemic exposure $\left(\mathrm{HbA}_{1 \mathrm{c}}\right)$ to the risk of development and progression of retinopathy in the Diabetes Control and Complications Trial. Diabetes 1995;4:968-83.

24 Warram JH, Manson JE, Krolewski AS. Glycosylated haemoglobin and the risk of retinopathy in insulindependent diabetes mellitus. $N$ Engl $\mathcal{F}$ Med 1995;332: 1305-6.

25 Early Treatment Diabetic Retinopathy Study Research Group. Effects of aspirin treatment on diabetic retinopathy. ETDRS Report Number 8. Ophthalmology 1991;98(Supp): 757-65.
26 Klein R, Klein BEK, Moss SE et al. The Wisconsin Epidemiologic Study of Diabetic Retinopathy. III. Prevalence miologic Study of Diabetic Retinopathy. III. Prevalence
and risk of diabetic retinopathy when age at diagnosis is 30 and risk of diabetic retinopathy when age at diagno
or more years. Arch Ophthalmol 1984;102:527-31.

27 National Health and Medical Research Council. Management of Diabetic Retinopathy. Clinical Practice Guidelines.

28 McCarty CA, Lloyd-Smith CW, Lee SE, et al. Use of eye care services by people with diabetes: the Melbourne Visual Impairment Project. Br f Ophthalmol 1998;82:410-414

29 Dickson PR, McCarty CA, Keeffe JE, et al. Diabetic retinopathy: examination practices and referral patterns of general practitioners. Med $\mathcal{F}$ Aust 1996;164:341-4.

30 Colagiuri S, Colagiuri R, Ward J. National diabetes strategy and implementation plan. Canberra: Diabetes Australia, 1998. 